\title{
The evolution of the small $x$ gluon TMD
}

\section{Jian Zhou}

School of physics, Shandong University, Jinan, Shandong 250100, China

Key Laboratory of Particle Physics and Particle Irradiation (MOE), Shandong University, Jinan, Shandong 250100, China

Nikhef,

De Boelelaan 1081, NL-1081 HV Amsterdam, The Netherlands

Department of Physics and Astronomy, VU University Amsterdam, De Boelelaan 1081, NL-1081 HV Amsterdam, The Netherlands

E-mail: jzhou@sdu.edu.cn

ABSTRACT: We study the evolution of the small $x$ gluon transverse momentum dependent (TMD) distribution in the dilute limit. The calculation has been carried out in the Ji-MaYuan scheme using a simple quark target model. As expected, we find that the resulting small $x$ gluon TMD simultaneously satisfies both the Collins-Soper (CS) evolution equation and the Balitsky-Fadin-Kuraev-Lipatov (BFKL) evolution equation. We thus confirmed the earlier finding that the high energy factorization (HEF) and the TMD factorization should be jointly employed to resum the different type large logarithms in a process where three relevant scales are well separated.

KeYwords: NLO Computations, QCD Phenomenology

ArXIV EPRINT: 1603.07426 


\section{Contents}

1 Introduction 1

2 The evolution of the small $x$ gluon TMD 2

2.1 Real corrections 4

$\begin{array}{lll}2.2 & \text { Virtual corrections } & 7\end{array}$

2.3 The gluon TMD at NLO in the leading logarithms approximation 9

$\begin{array}{lll}3 & \text { Summary } & 10\end{array}$

\section{Introduction}

QCD factorization theorems are important tools in describing hard processes of the strong interaction. Among many factorization frameworks, transverse momentum dependent (TMD) factorization [1-3] in terms of the Collins-Soper evolution equation [1, 2] or high energy factorization (HEF) $[4,5]$ in terms of the BFKL evolution equation $[6,7]$ should be employed when computing an observable that is sensitive to parton transverse momentum in high energy scatterings. These two factorization frameworks are applicable in the different kinematical regions. For example, in the process of the color neutral scalar particle production through gluon fusion $(g g \rightarrow H)$ in proton proton collisions, the HEF is valid in the kinematical region $S \gg M^{2}$ where $S$ is the center mass of energy squared and $M$ is the scalar particle mass, while the TMD factorization holds as long as $p_{\perp}^{2} \ll M^{2}$ with $p_{\perp}$ being the produced scalar particle transverse momentum.

Apparently, there exists an overlap region $S \gg M^{2} \gg p_{\perp}^{2}$ where the both factorization formalisms can apply. The natural questions that would be asked then are: do they become equivalent and produce the same result in the overlap region; or are they complementary to each other in a such region? A recent study [8] suggests that the later one is a right question in the sense that the large logarithm terms $\ln \frac{S}{M^{2}}$ and $\ln \frac{M^{2}}{p_{\perp}^{2}}$ show up at higher orders need to be simultaneously resumed in the small $x$ formalism and the TMD factorization framework respectively. To be more specific, the large logarithm $\ln \frac{S}{M^{2}}$ in the dilute limit can be resummed by means of the BFKL equation which governs the rapidity evolution of the unintegrated gluon distribution appears in the HEF formula, while the large logarithm $\ln \frac{M^{2}}{p_{\perp}^{2}}$ can be conveniently taken care by the Collins-Soper equation that describes the energy dependence of the gluon TMD appears in the TMD factorization cross section.

In refs. $[8,9]$, an explicit next to leading order (NLO) calculation for the scalar particle production process has been performed in the small $x$ formalism (the color glass condensate effective theory $[11-13,13,14]$ ) which is reduced to the HEF in the dilute limit. It has been found that the two different type logarithms can be resummed consistently at the same 
time. The critical step of achieving this is to realize that the the logarithms $\ln \frac{S}{M^{2}}$ and $\ln \frac{M^{2}}{p_{\perp}^{2}}$ receive contributions from the clearly separated phase space regions of the radiated gluon. This is because the rapidity divergence comes from the strong rapidity ordering region and the soft gluon is responsible for the light cone divergence in the double leading logarithm approximation. These analysis have been extended to the other processes, including heavy quark pair production, back-to-back di-jet production in $e A$ and $p A$ collisions, and MuellerNavelet Dijet production $[9,10]$.

Inspired by refs. $[8,9]$, we address the same topic from a different aspect of view in this paper. Specifically, we do not attempt to extract the large logarithm contributions from the complete NLO result for a cross section. Instead, we assume that the unintegrated gluon distribution and the gluon TMD are essentially the same object and share the same operator definition in the overlap region. One then can compute the LO and NLO contributions to the gluon TMD/unintegrated gluon distribution starting from their operator definition in

a simple quark target model. We observed that two different logarithms $\ln \frac{1}{x}$ and $\ln \frac{x^{2} \zeta^{2}}{l_{\perp}^{2}}$ show up simultaneously at the NLO, where $l_{\perp}$ is the gluon transverse momentum and $\zeta$ is a parameter proportional to the plus momentum of hadron. When embedding the gluon TMD/unintegrated gluon distribution into a cross section formula, these two logarithms will be converted into the $\operatorname{logarithms} \ln \frac{S}{M^{2}}$ and $\ln \frac{M^{2}}{p_{\perp}^{2}}$. Furthermore, we found that the dependencies of the gluon TMD/unintegrated gluon distribution on the logarithms of $x$ and $\zeta$ are controlled by the BFKL and CS equation, respectively. In addition, it has been confirmed that the BFKL equation and the CS evolution are driven by radiated gluons from the different phase space regions. As the first step, we restrict us to the dilute limit and do not take into account any non-linear saturation effect $[12,13,13-16]$ in this work.

The interplay of TMD/spin physics and small $x$ physics is becoming a topical issue in recent years $[8,9,17-43,47]$. In particular, we notice that the attempts to unify the description of various types evolution of the gluon TMD have also been made in refs. [28, 3943]. However, the calculations presented in refs. [28, 39-43] are formulated in the very different ways. It is a non-trivial task to compare the current work with these formalisms. We thus leave this for the future study. We also would like to mention that the other type joint resummation has been discussed in the literatures [44-46].

The rest of the paper is structured as follows: in the next section, we calculate the next to leading order correction to the gluon TMD in the small $x$ limit and check if the resulting small $x$ gluon TMD satisfies the both BFKL and CS evolution equations; in the section III, we comment on the implications of our result and outline the possible extensions/generalizations of the current work.

\section{The evolution of the small $x$ gluon TMD}

In this section, we calculate the next to leading order correction to the gluon TMD in a simple quark target model and show that the derived gluon TMD satisfies both the CS equation and the BFKL equation simultaneously. Our starting point is the matrix element 
definition for the unpolarized gluon TMD/unintegrated gluon distribution $[1,2,48,50]$,

$x G\left(x, l_{\perp}, x \zeta\right)=\int \frac{d y^{-} d^{2} y_{\perp}}{(2 \pi)^{3} P^{+}} e^{-i x P^{+} y^{-}+i l_{\perp} \cdot y_{\perp}}\left\langle P\left|F_{\mu}^{+}\left(y^{-}, y_{\perp}\right) \mathcal{L}_{\tilde{n}}^{\dagger}\left(y^{-}, y_{\perp}\right) \mathcal{L}_{\tilde{n}}\left(0,0_{\perp}\right) F^{\mu+}(0)\right| P\right\rangle$

where $\mathcal{L}$ is the process dependent gauge link. In this work, we perform the calculation using a future pointing staple like gauge link,

$$
\mathcal{L}\left(\infty ; y^{-}, y_{\perp}\right)=\mathcal{P} \exp \left(-i g \int_{0}^{\infty} d \lambda \tilde{n} \cdot A\left(\lambda \tilde{n}+y^{-}, y_{\perp}\right)\right)
$$

Here $A^{\mu}=-i f_{a b c} A_{c}^{\mu}$ is the gluon potential in the adjoint representation. In the Ji-MaYuan scheme [49, 50] (or the old Collins-Soper scheme [1, 2]), $\tilde{n}$ is defined as a slightly off-light-cone vector $\tilde{n}=n+\delta^{+} p$ where $0<\delta^{+} \ll 1$ is a small parameter, and $n=$ $\left(1,0,0_{\perp}\right), p=\left(0,1,0_{\perp}\right)$ are the commonly defined light cone vectors. With a non-light-like vector $\tilde{n}$, the gluon TMD depends on a new scalar $\zeta^{2}=2\left(P^{+}\right)^{2} / \delta^{+}$, where $P^{+}$is the nucleon plus momentum. Apart from the $\zeta$ dependence, the gluon TMD also depend on the renormalization scale $\mu$, that is not discussed here as it is beyond the scope of this work. Note that a soft factor in the properly defined gluon TMDs is omitted in the above definition. This does not affect the result of the current work because the soft factor in the Ji-Ma-Yuan scheme is independent of the parameter $\zeta$. Consequently, the subtracted TMDs and the un-subtracted TMDs in the Ji-Ma-Yuan scheme satisfy the same CollinsSoper evolution equation.

Now we briefly explain why one needs to introduce $\zeta$ dependence by investigating the singularity of the DGLAP splitting kernel from the real diagrams contributions,

$$
\mathcal{P}_{g g}(z)=2 C_{A} \frac{\left(z^{2}-z+1\right)^{2}}{z(1-z)}
$$

where $z=x / x^{\prime}$ is the longitudinal momentum fraction of parent gluon carried by the outgoing gluon. It is easy to see that such splitting kernel is divergent when $z$ approaches 1 . This divergence is often referred to as the light cone divergence which originates from the contributions of real gluons emitted from gauge links with zero plus momentum. In the case of the integrated gluon distribution (normal gluon PDF), the light cone divergence is canceled by the virtual corrections. However, such cancelation does not occur locally if we leave the gluon transverse momentum $l_{\perp}$ unintegrated. A few ways of regulating the lightcone singularity have been put forward [49-54], among which the Ji-Ma-Yuan scheme [49] turns out to be the most convenient choice for the current purpose of this work. In a such scheme, a large logarithm $\ln \frac{x^{2} \zeta^{2}}{l_{\perp}^{2}}$ contribution to the gluon TMD will emerge and can be summed to all orders utilizing the CS evolution equation.

On the other hand, it is easy to see that the splitting function are singular when $x$ (or $z$ ) approaches zero. The large logarithm $\ln \frac{1}{x}$ contribution become important in this kinematical limit. Resummation of this large logarithms in the linear approximation (or in the dilute limit) is accomplished by the BFKL evolution equation.

In the present paper, we show that the resummation of the two different type large logarithms can be done in an unified framework. To this end, we first compute the leading 


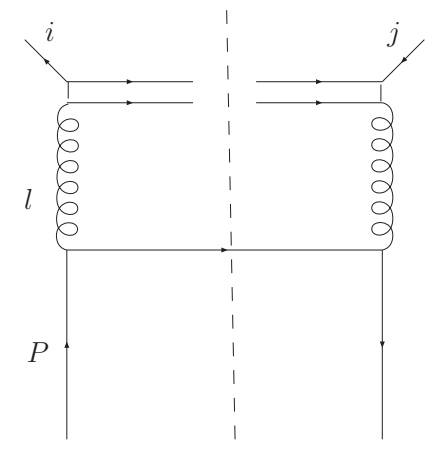

Figure 1. The leading order diagram contributing to the gluon TMD in the quark target model. $P$ and $l$ are the incoming quark and the outgoing gluon momenta, respectively. $i, j$ denote the gluon polarization indices which will be contracted with $\delta_{\perp}^{i j}$ in the unpolarized case.

order contribution to the gluon TMD in a simple quark target model. We then proceed to study how the gluon TMD is dressed by the quantum corrections at the next to leading order. With the computed gluon TMD at NLO, we will verify that it satisfies the both CS evolution equation and BFKL evolution equation.

In the quark target model, the leading order contribution to the gluon TMD only comes from one graph illustrated in the figure 1 and is given by,

$$
G\left(x, l_{\perp}, x \zeta\right)_{L O}=\frac{\alpha_{s} C_{F}}{2 \pi^{2}} \frac{1}{l_{\perp}^{2}} \frac{1+(1-x)^{2}}{x}
$$

where $x=l^{+} / P^{+}$with $P^{+}$and $l^{+}$being the incoming quark and the outgoing gluon momenta respectively. In the small $x$ limit, it is simplified as,

$$
\left.x G\left(x, l_{\perp}, x \zeta\right)_{L O}\right|_{x \rightarrow 0}=\frac{\alpha_{s} C_{F}}{\pi^{2}} \frac{1}{l_{\perp}^{2}}
$$

Here one immediately notices that the expression for the gluon TMD at the leading order contains neither the logarithm $\ln \frac{1}{x}$ nor the logarithm $\ln \frac{x^{2} \zeta^{2}}{l_{\perp}^{2}}$. It is thus necessary to extend the analysis to the next to leading order for the purpose of studying the evolution of small $x$ gluon TMD.

\subsection{Real corrections}

In this subsection, we calculate the NLO real corrections to the gluon TMD. At NLO, there are a number of diagrams contributing to the gluon TMD, some of which have been shown in the figure 2. Among these real graphs, the figure 2(a) is a particularly interesting one, because the figure 2(a) and its conjugate diagrams are the only real diagrams contributing to the both evolution kernels of the BFKL and the CS equations in a covariant gauge calculation. It would be instructive to present some technical steps for the calculation of the figure $2(\mathrm{a})$. 


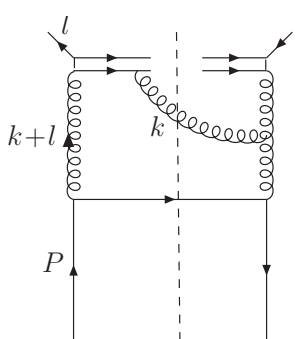

(a)

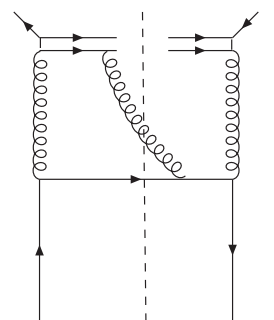

(b)

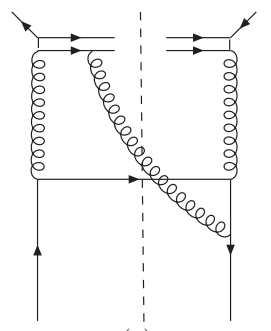

(c)

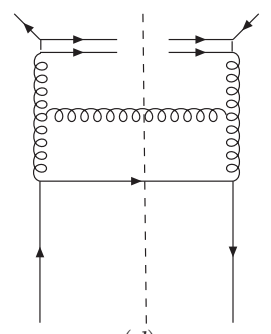

(d)

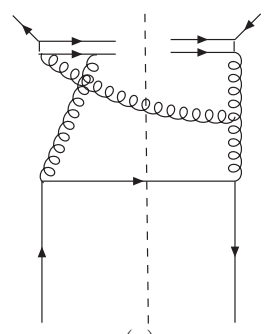

(e)

Figure 2. Sample real diagrams contributing to the evolution kernels of the CS equation and the BFKL equation. All real diagrams shown here contribute to the evolution kernel of the BFKL equation. The figure (a) and its conjugate diagram are the only real corrections that contribute to both the evolution kernels of the BFKL equation and the CS equation.

The calculation of the real correction from the figure 2(a) involves the following integration,

$$
\begin{aligned}
I_{2(a)}= & \mathcal{C} \int d l^{-} d^{4} k \delta\left((P-l-k)^{2}\right) \delta\left(k^{2}\right) \theta\left(k^{+}\right) \theta\left(P^{+}-l^{+}-k^{+}\right) \\
& \times \frac{2\left(P^{+}-l^{+}-k^{+}\right)\left[k^{+}\left(k_{\perp}+l_{\perp}\right) \cdot l_{\perp}-2 l^{+}\left(l_{\perp}+k_{\perp}\right)^{2}\right]}{\left[(k+l)^{2}+i \epsilon\right]^{2}\left[k^{+}+\delta^{+} k^{-}+i \epsilon\right]\left[l^{2}+i \epsilon\right]}
\end{aligned}
$$

where $\mathcal{C}=\alpha_{s}^{2}\left(N_{c}^{2}-1\right) / 4 \pi^{4}$. The radiated gluon momentum is denoted as $k$. The first step is to carry out the integration over $l^{-}$and $k^{-}$using the delta function which comes from the on shell condition. After integrating out $l^{-}$and $k^{-}$, one has the kinematical constraints $l^{-}+k^{-}=-\left(l_{\perp}+k_{\perp}\right)^{2} / 2\left(P^{+}-l^{+}-k^{+}\right)$and $k^{-}=k_{\perp}^{2} / 2 k^{+}$. To simplify the calculation, we make the approximation $-l^{-} \approx k^{-}=\frac{k_{\perp}^{2}}{2 k^{+}}$that is valid in the small $x$ region, and obtain,

$$
I_{2(a)}=-\mathcal{C} \int d^{2} k_{\perp} \int_{0}^{P^{+}} d k^{+} \frac{k^{+}\left[k^{+}\left(k_{\perp}+l_{\perp}\right) \cdot l_{\perp}-2 l^{+}\left(l_{\perp}+k_{\perp}\right)^{2}\right]}{\left(k_{\perp}+l_{\perp}\right)^{4}\left[2\left(k^{+}\right)^{2}+\delta^{+} k_{\perp}^{2}\right]\left[l^{+} k_{\perp}^{2}+k^{+} l_{\perp}^{2}\right]}
$$

We proceed by separating $k^{+}$integration into two parts $\int_{0}^{P^{+}} d k^{+}=\int_{0}^{l^{+}} d k^{+}+\int_{l^{+}}^{P^{+}} d k^{+}$. Integrating out $k^{+}$and keeping only the large logarithm terms, one arrives,

$$
\begin{aligned}
I_{2(a)}= & \mathcal{C} \int d^{2} k_{\perp} \frac{1}{\left(k_{\perp}+l_{\perp}\right)^{2} 2\left[k_{\perp}^{2}+l_{\perp}^{4} / x^{2} \zeta^{2}\right]} \ln \frac{k_{\perp}^{2}\left(k_{\perp}^{2}+x^{2} \zeta^{2}\right)}{\left(k_{\perp}^{2}+l_{\perp}^{2}\right)^{2}}+\mathcal{O}\left(\frac{1}{\ln \frac{x^{2} \zeta^{2}}{l_{\perp}^{2}}}\right) \\
& -\mathcal{C} \ln \frac{1}{x} \int_{0} d^{2} k_{\perp} \frac{\left(k_{\perp}+l_{\perp}\right) \cdot l_{\perp}}{\left(k_{\perp}+l_{\perp}\right)^{4}} \frac{1}{2 l_{\perp}^{2}}+\mathcal{O}\left(\frac{1}{\ln \frac{1}{x}}\right)
\end{aligned}
$$

where it is easy to see that two different type logarithms arise from the clearly separated phase space regions. To be more precise, the Collins-Soper type evolution is driven by the emitted gluon which carries very small longitudinal momentum $k^{+} \ll l^{+}$. In contrast, the BFKL evolution kernel only receives the contribution from the phase space region where $k^{+} \gg l^{+}$, the so-called strong rapidity ordering region. This finding is consistent with the observation made in ref. [8]. Note that this statement is not necessarily true for the 


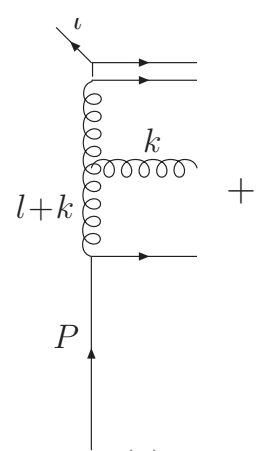

(a)

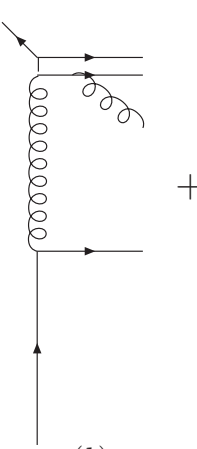

(b)

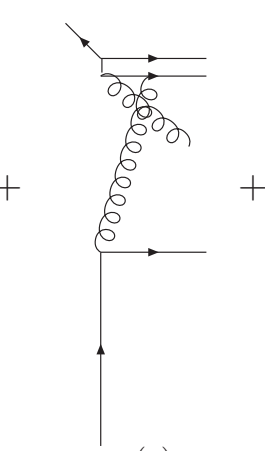

(c)

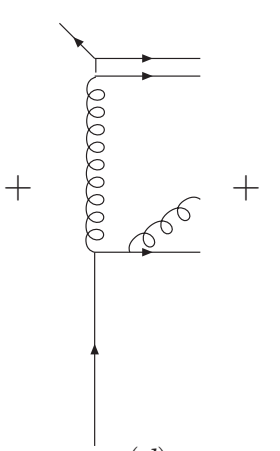

(d)

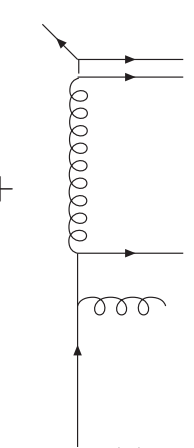

(e)

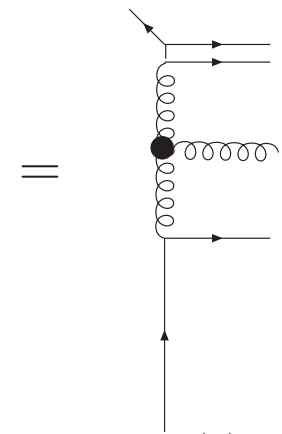

(f)

Figure 3. In the strong rapidity ordering region $l^{+} \ll k^{+} \ll P^{+}$, all real corrections figure (a)figure (e) can be summarized into one diagram figure (f) with an effective Lipatov vertex. The large solid circle denotes the Lipatov vertex.

Sudakov single leading logarithm term which also receives the contribution from the hard gluon emissions in the virtual correction case.

At this step, we have completed the calculation of the real correction to the CS evolution kernel. However, as mentioned earlier, many other real diagrams give rise to the large $\operatorname{logarithm} \ln \frac{1}{x}$ contribution as well. Instead of calculating them one by one by brute force, we simplify the calculation by isolating the leading logarithm contribution with the help of the so-called effective Lipatov vertex. It is well known that the leading logarithms of $x$ in the BFKL dynamics are generated in the strong rapidity ordering region: $P^{+} \gg k^{+} \gg l^{+}$. In a such kinematical region, it is justified to approximate the incoming and outgoing quark lines as the Eikonal lines. After making this approximation, as illustrated in the figure 3, graphs with all possible gluon insertions can be summarized into one diagram with an effective Liaptov vertex. The effective Lipatov vertex reads,

$$
C^{\mu}=g\left\{\left(k_{T}+2 l_{T}\right)^{\mu}-\left[k^{+}-\frac{\left(k_{\perp}+l_{\perp}\right)^{2}}{k^{-}}\right] p^{\mu}+\left[k^{-}-\frac{l_{\perp}^{2}}{k^{+}}\right] n^{\mu}\right\}
$$

where $k_{T}$ is the transverse component of four momentum with $k_{T}^{2}=-k_{\perp}^{2}$. Such an effective vertex is gauge invariant and satisfies $C_{\mu} k^{\mu}=0$. By simply squaring the amplitude and using the relation $C^{\mu} C_{\mu}=g^{2} \frac{-4\left(k_{\perp}+l_{\perp}\right)^{2} l_{\perp}^{2}}{k_{\perp}^{2}}$, it is straightforward to calculate the real gluon contribution to the logarithm $\ln \frac{1}{x}$ terms. The total real correction to the gluon TMD in the leading logarithms approximation is then given by,

$$
\begin{aligned}
\left.x G\left(x, l_{\perp}, x \zeta\right)_{R e l}\right|_{x \rightarrow 0}= & \mathcal{C} \int d^{2} k_{\perp} \frac{1}{\left(k_{\perp}+l_{\perp}\right)^{2}\left[k_{\perp}^{2}+l_{\perp}^{4} / x^{2} \zeta^{2}\right]} \ln \frac{k_{\perp}^{2}\left(k_{\perp}^{2}+x^{2} \zeta^{2}\right)}{\left(k_{\perp}^{2}+l_{\perp}^{2}\right)^{2}} \\
& +2 \mathcal{C} \ln \frac{1}{x} \int_{0} \frac{d^{2} k_{\perp}}{\left(k_{\perp}+l_{\perp}\right)^{2} k_{\perp}^{2}}
\end{aligned}
$$

Using the eq. (1), the NLO real correction to the gluon TMD can be reexpressed in terms 


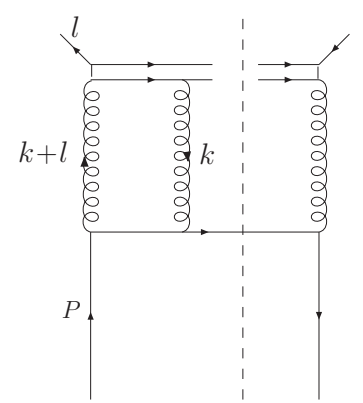

(a)

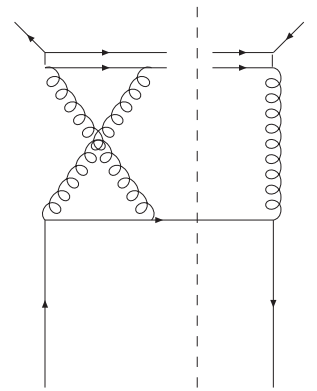

(b)

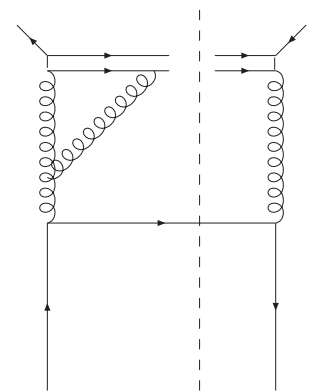

(c)

Figure 4. Virtual diagrams contributing to the gluon TMD at NLO. The figure (a) and figure (b) and their conjugate diagrams give rise to the virtual corrections to the BFKL evolution kernel, while the figure (c) and its conjugate diagram contribute to the CS evolution kernel.

of the leading order gluon TMD,

$$
\left.x G\left(x, l_{\perp}, x \zeta\right)_{R e l}\right|_{x \rightarrow 0}=\frac{\alpha_{s} N_{c}}{\pi^{2}} \int d^{2} k_{\perp}\left\{\frac{\ln \frac{1}{x}}{k_{\perp}^{2}}+\frac{\ln \frac{k_{\perp}^{2}\left(k_{\perp}^{2}+x^{2} \zeta^{2}\right)}{\left(k_{\perp}^{2}+l_{\perp}^{2}\right)^{2}}}{2\left[k_{\perp}^{2}+l_{\perp}^{4} / x^{2} \zeta^{2}\right]}\right\} x G_{L O}\left(x, k_{\perp}+l_{\perp}, x \zeta\right)
$$

\section{$2.2 \quad$ Virtual corrections}

We now move on to evaluate virtual corrections. Among many virtual diagrams, the figure 4(a), figure 4(b) and their conjugate diagrams produce the large logarithm of $x$ [55, 56]. On the other hand, the light cone divergence is only generated from the figure 4(c) and its mirror diagram.

Let us first focus on isolating the leading logarithm of $x$ from the figure 4 (a), whose contribution reads

$$
\begin{aligned}
I_{4(a)}= & \frac{-i \mathcal{C}}{4 \pi} \int d l^{-} \int d^{4} k \\
& \times \frac{4\left(P^{+}-l^{+}\right)\left(P^{+}-k^{+}-l^{+}\right)\left[\left(k_{\perp}+l_{\perp}\right) \cdot l_{\perp}\right]}{\left[(k+l)^{2}+i \epsilon\right]\left[k^{2}+i \epsilon\right]\left[(P-k-l)^{2}+i \epsilon\right]\left[k^{+}+i \epsilon\right]\left[l^{2}+i \epsilon\right]} \delta\left((P-l)^{2}\right)
\end{aligned}
$$

where the Eikonal approximation has been applied to the quark line. In the above formula, we do not regularize the gauge link propagator as this integral is free from the light cone singularity. It is straightforward to carry out $l^{-}$integration using the delta function,

$$
\begin{aligned}
I_{4(a)}= & \frac{-i \mathcal{C}}{4 \pi} \int d^{4} k \frac{2\left(P^{+}-k^{+}-l^{+}\right)}{\left[2\left(k^{+}+l^{+}\right) k^{-}-\left(k_{\perp}+l_{\perp}\right)^{2}+i \epsilon\right]\left[2 k^{+} k^{-}-k_{\perp}^{2}+i \epsilon\right]} \\
& \times \frac{\left[\left(k_{\perp}+l_{\perp}\right) \cdot l_{\perp}\right]}{\left[-2\left(P^{+}-k^{+}-l^{+}\right) k^{-}-\left(k_{\perp}+l_{\perp}\right)^{2}+i \epsilon\right]\left[k^{+}+i \epsilon\right]\left[l_{\perp}^{2}\right]}
\end{aligned}
$$

Here and hereafter, we ignore $l^{-} \approx-l_{\perp}^{2} / 2 P^{+}$that appears in the integral. We then proceed 
to do the $k^{-}$integration by closing different contours depending on the value of $k^{+}$,

$$
\begin{aligned}
I_{4(a)}= & \frac{\mathcal{C}}{2}\left\{\int_{-l^{+}}^{0} d k^{+} d^{2} k_{\perp} \frac{\left(k_{\perp}+l_{\perp}\right) \cdot l_{\perp}}{\left(k_{\perp}+l_{\perp}\right)^{2} l_{\perp}^{2}} \frac{k^{+}+l^{+}}{\left[k^{+}\left(k_{\perp}+l_{\perp}\right)^{2}-\left(k^{+}+l^{+}\right) k_{\perp}^{2}+i \epsilon\right]} \frac{1}{\left[k^{+}+i \epsilon\right]}\right. \\
& \left.+\int_{0}^{P^{+}-l^{+}} d k^{+} d^{2} k_{\perp} \frac{\left(k_{\perp}+l_{\perp}\right) \cdot l_{\perp}}{\left(k_{\perp}+l_{\perp}\right)^{2} l_{\perp}^{2}} \frac{P^{+}-k^{+}-l^{+}}{\left[k^{+}\left(k_{\perp}+l_{\perp}\right)^{2}-\left(P^{+}-k^{+}-l^{+}\right) k_{\perp}^{2}+i \epsilon\right]} \frac{1}{\left[k^{+}+i \epsilon\right]}\right\}
\end{aligned}
$$

One notices that each of two integrals contains the light cone singularity when $k^{+}$approaches zero. However, the sum of them is free from the light cone divergence and is given by,

$$
\begin{aligned}
I_{4(a)}= & \frac{\mathcal{C}}{2} \int d^{2} k_{\perp} \frac{\left(k_{\perp}+l_{\perp}\right) \cdot l_{\perp}}{\left(k_{\perp}+l_{\perp}\right)^{2} l_{\perp}^{2} k_{\perp}^{2}} \\
& \times\left[\ln \frac{l^{+}}{P^{+}-l^{+}}+\frac{2 k_{\perp}^{2}\left(k_{\perp}+l_{\perp}\right)^{2}}{\left(k_{\perp}+l_{\perp}\right)^{4}-k_{\perp}^{4}} \ln \frac{k_{\perp}^{2}}{\left(k_{\perp}+l_{\perp}\right)^{2}}+i \pi \frac{k_{\perp}^{2}+2\left(k_{\perp}+l_{\perp}\right)^{2}}{k_{\perp}^{2}+\left(k_{\perp}+l_{\perp}\right)^{2}}\right]
\end{aligned}
$$

where the leading logarithm term can be further re-expressed as,

$$
I_{4(a)}=-\frac{\mathcal{C}}{4} \ln \frac{1}{x} \int d^{2} k_{\perp} \frac{1}{k_{\perp}^{2}} \frac{1}{\left(k_{\perp}+l_{\perp}\right)^{2}}+\mathcal{O}\left(\frac{1}{\ln \frac{1}{x}}\right)
$$

In arriving at the above result, we have made use of the following identity,

$$
\int d^{2} k_{\perp} \frac{\left(k_{\perp}+l_{\perp}\right) \cdot l_{\perp}}{k_{\perp}^{2}\left(k_{\perp}+l_{\perp}\right)^{2}}=-\int d^{2} k_{\perp} \frac{k_{\perp} \cdot l_{\perp}}{k_{\perp}^{2}\left(k_{\perp}+l_{\perp}\right)^{2}}
$$

which can be easily verified by changing the integration variable $k_{\perp} \rightarrow-k_{\perp}-l_{\perp}$. Repeating the similar procedure, for the figure 4(b), one obtains,

$$
I_{4(b)}=-\frac{\mathcal{C}}{4} \ln \frac{1}{x} \int d^{2} k_{\perp} \frac{1}{k_{\perp}^{2}} \frac{1}{\left(k_{\perp}+l_{\perp}\right)^{2}}+\mathcal{O}\left(\frac{1}{\ln \frac{1}{x}}\right)
$$

To complete the calculation of virtual corrections in the leading logarithm approximation, we also need include the contribution from the figure 4(c),

$$
I_{4(c)}=\frac{i \mathcal{C} \mu^{2 \epsilon}}{(2 \pi)^{1-2 \epsilon}} \int d l^{-} \int d^{4-2 \epsilon} k \frac{2\left(P^{+}-l^{+}\right)\left[2\left(l^{+}+k^{+}\right) l_{\perp}^{2}+2 k^{+} l_{\perp} \cdot k_{\perp}\right]}{\left[(k+l)^{2}+i \epsilon\right]\left[k^{2}+i \epsilon\right]\left[k^{+}+\delta^{+} k^{-}+i \epsilon\right]\left[l^{2}+i \epsilon\right]^{2}} \delta\left((P-l)^{2}\right)
$$

where we use dimensional regularization to treat ultraviolet divergences. It is again convenient to first carry out the integration over $l^{-}$and $k^{-}$. One obtains,

$$
\begin{aligned}
I_{4(c)}= & \frac{\mathcal{C} \mu^{2 \epsilon}}{(2 \pi)^{-2 \epsilon}}\left\{\int_{-l^{+}}^{0} d k^{+} \int d^{2-2 \epsilon} k_{\perp} \frac{k^{+}\left[2\left(l^{+}+k^{+}\right) l_{\perp}^{2}+2 k^{+} l_{\perp} \cdot k_{\perp}\right]}{\left[\left(k^{+}+l^{+}\right) k_{\perp}^{2}-k^{+}\left(k_{\perp}+l_{\perp}\right)^{2}\right]\left[2\left(k^{+}\right)^{2}+\delta^{+} k_{\perp}^{2}\right]\left[l_{\perp}^{4}\right]}\right. \\
& \left.+\int_{-\infty}^{-l^{+}} d k^{+} \int d^{2-2 \epsilon} k_{\perp} \frac{\delta^{+}\left[2\left(l^{+}+k^{+}\right) l_{\perp}^{2}+2 k^{+} l_{\perp} \cdot k_{\perp}\right]}{\left[2\left(k^{+}+l^{+}\right) k^{+}+\delta^{+}\left(k_{\perp}+l_{\perp}\right)^{2}\right]\left[2\left(k^{+}\right)^{2}+\delta^{+} k_{\perp}^{2}\right]\left[l_{\perp}^{4}\right]}\right\}
\end{aligned}
$$


Here $l^{-} \approx-l_{\perp}^{2} / 2 P^{+}$component has been neglected. It is easy to further integrate out $k_{\perp}$ by applying the Feynman parametrization. We are then only left with the integration with respect to $k^{+}$and the Feynman parameter. At this step, though it is still hard to get the complete analytical result, one can readily extract the double leading logarithm and the single leading logarithm contributions,

$$
I_{4(c)}=\frac{\mathcal{C} \pi}{2 l_{\perp}^{2}}\left[\frac{1}{2 \epsilon}-\frac{1}{2} \ln \frac{x^{2} \zeta^{2}}{\mu^{2}}+\ln \frac{x^{2} \zeta^{2}}{l_{\perp}^{2}}-\left(\ln \frac{x^{2} \zeta^{2}}{l_{\perp}^{2}}\right)^{2}\right]+\mathcal{O}\left(\frac{1}{\ln \frac{x^{2} \zeta^{2}}{l_{\perp}^{2}}}\right)
$$

where the ultraviolet pole can be simply removed according to the minimal subtraction scheme. One notices that the off-shellness of the incoming gluon serves a natural infrared cut off. At this point, it is worthy mentioning that the double leading logarithm term in the above formula only receives the contribution from the phase space region where $\left|k^{+}\right| \ll\left|l^{+}\right|$which is beyond the scope of the strong rapidity ordering region. Collecting all contributions from the figure 4(a), figure 4(b), figure 4(c) and their mirror graphs together, the total virtual correction to the small $x$ gluon TMD is given by,

$$
\left.x G\left(x, l_{\perp}, x \zeta\right)_{V i r}\right|_{x \rightarrow 0}=-\mathcal{C} \ln \frac{1}{x} \int \frac{d^{2} k_{\perp}}{\left(k_{\perp}+l_{\perp}\right)^{2} k_{\perp}^{2}}+\frac{\mathcal{C} \pi}{l_{\perp}^{2}}\left[\ln \frac{x^{2} \zeta^{2}}{l_{\perp}^{2}}-\frac{1}{2} \ln \frac{x^{2} \zeta^{2}}{\mu^{2}}-\left(\ln \frac{x^{2} \zeta^{2}}{l_{\perp}^{2}}\right)^{2}\right]
$$

Using eq. (2.5), it can be re-expressed as,

$$
\begin{aligned}
x G\left(x, l_{\perp}, x \zeta\right)_{N L O}= & \frac{\alpha_{s} N_{c}}{2 \pi}\left[\ln \frac{x^{2} \zeta^{2}}{l_{\perp}^{2}}-\frac{1}{2} \ln \frac{x^{2} \zeta^{2}}{\mu^{2}}-\left(\ln \frac{x^{2} \zeta^{2}}{l_{\perp}^{2}}\right)^{2}\right] x G_{L O}\left(x, l_{\perp}, x \zeta\right) \\
& -\frac{\alpha_{s} N_{c}}{2 \pi^{2}} \ln \frac{1}{x} \int \frac{d^{2} k_{\perp}}{k_{\perp}^{2}} \frac{l_{\perp}^{2}}{\left(l_{\perp}+k_{\perp}\right)^{2}} x G_{L O}\left(x, l_{\perp}, x \zeta\right)
\end{aligned}
$$

\subsection{The gluon TMD at NLO in the leading logarithms approximation}

Combining the real correction and the virtual correction obtained in the previous subsections, up to the leading logarithms accuracy the gluon TMD at NLO is given by,

$$
x G\left(x, l_{\perp}, x \zeta\right)_{N L O}=x G\left(x, l_{\perp}, x \zeta\right)_{L O}+x G\left(x, l_{\perp}, x \zeta\right)_{R e l}+x G\left(x, l_{\perp}, x \zeta\right)_{V i r}
$$

The gluon TMD at NLO in the small $x$ then reads,

$$
\begin{aligned}
x G\left(x, l_{\perp}, x \zeta\right)_{N L O} & =x G\left(x, l_{\perp}, x \zeta\right)_{L O} \\
& +\frac{\alpha_{s} N_{c}}{\pi^{2}} \ln \frac{1}{x} \int \frac{d^{2} k_{\perp}}{k_{\perp}^{2}}\left[x G_{L O}\left(x, k_{\perp}+l_{\perp}, x \zeta\right)-\frac{l_{\perp}^{2}}{2\left(l_{\perp}+k_{\perp}\right)^{2}} x G_{L O}\left(x, l_{\perp}, x \zeta\right)\right] \\
& +\frac{\alpha_{s} N_{c}}{2 \pi}\left[\ln \frac{x^{2} \zeta^{2}}{l_{\perp}^{2}}-\frac{1}{2} \ln \frac{x^{2} \zeta^{2}}{\mu^{2}}-\left(\ln \frac{x^{2} \zeta^{2}}{l_{\perp}^{2}}\right)^{2}\right] x G_{L O}\left(x, l_{\perp}, x \zeta\right) \\
& +\frac{\alpha_{s} N_{c}}{\pi^{2}} \int \frac{d^{2} k_{\perp}}{2\left[k_{\perp}^{2}+l_{\perp}^{4} / x^{2} \zeta^{2}\right]} \ln \frac{k_{\perp}^{2}\left(k_{\perp}^{2}+x^{2} \zeta^{2}\right)}{\left(k_{\perp}^{2}+l_{\perp}^{2}\right)^{2}} x G_{L O}\left(x, k_{\perp}+l_{\perp}, x \zeta\right)
\end{aligned}
$$

This is the main result of our paper. 
We are now ready to check if the computed small $x$ gluon TMD at NLO satisfies the both BFKL and CS evolution equations. For the $\ln \frac{1}{x}$ dependence, it is easy to see that the resulting gluon TMD satisfies the following equation in the strong rapidity ordering region,

$$
\frac{\partial\left[x G\left(x, l_{\perp}, x \zeta\right)\right]}{\partial \ln (1 / x)}=\frac{\alpha_{s} N_{c}}{\pi^{2}} \int \frac{d^{2} k_{\perp}}{k_{\perp}^{2}}\left\{x G\left(x, k_{\perp}+l_{\perp}, x \zeta\right)-\frac{l_{\perp}^{2}}{2\left(l_{\perp}+k_{\perp}\right)^{2}} x G\left(x, l_{\perp}, x \zeta\right)\right\}
$$

which is just the famous BFKL evolution equation. For the $\ln \zeta$ dependence, the derivative of the computed gluon TMD with respect to $\ln \zeta$ is given by,

$$
\begin{aligned}
\frac{\partial\left[G\left(x, l_{\perp}, x \zeta\right)\right]}{\partial \ln \zeta}= & \frac{\alpha_{s} N_{c}}{\pi^{2}} \int d^{2} k_{\perp} \frac{1}{k_{\perp}^{2}+l_{\perp}^{4} / x^{2} \zeta^{2}} G\left(x, k_{\perp}+l_{\perp}, x \zeta\right) \\
& +\frac{\alpha_{s} N_{c}}{\pi}\left[\frac{1}{2}-2 \ln \frac{x^{2} \zeta^{2}}{l_{\perp}^{2}}\right] G\left(x, l_{\perp}, x \zeta\right)
\end{aligned}
$$

where the terms suppressed by the power of $1 / \zeta^{2}$ have been neglected. In the impact parameter $b_{\perp}$ space, it takes a more conventional form,

$$
\frac{\partial\left[G\left(x, b_{\perp}, x \zeta\right)\right]}{\partial \ln \zeta}=-\frac{\alpha_{s} N_{c}}{\pi} \ln \left[\frac{x^{2} \zeta^{2} b_{\perp}^{2}}{4} e^{2 \gamma_{E}-\frac{1}{2}}\right] G\left(x, b_{\perp}, x \zeta\right)
$$

which can be recognized as the famous CS evolution equation for the gluon TMD. However, our result differs from that derived in ref. [50] by a finite term. More work will be needed to settle down this issue as we are not able to localize the source of this discrepancy for the time being. Nevertheless, based on the above findings, it might be fair to claim that it is possible to describe the BFKL and CS dynamics in an unified framework.

\section{Summary}

We end this paper with a short summary of our major results and a number of remarks on their implications. We compute the NLO correction to the small $x$ gluon TMD in the leading logarithm approximation starting from the well known operator definition of the gluon TMD. It is shown that the resulting gluon TMD simultaneously satisfies the both CS equation and BFKL equation. One thus may conclude that the unintegrated gluon distribution and the gluon TMD share the same operator definition in the overlap region where both the TMD factorization and the HEF apply. Moreover, we confirmed the observation made in refs. $[8,9]$ that the BFKL evolution kernel and the double leading logarithm part of the CS evolution kernel receive contributions from the clearly separated phase space regions. To be more specific, the double leading logarithm contribution to the CS evolution is yielded from the phase space region outside the scope of the strong rapidity ordering region.

In order to resum two different type but equally important logarithms $\ln \frac{S}{M^{2}}$ and $\ln \frac{M^{2}}{p_{\perp}^{2}}$ in the overlap region which is $S \gg M^{2} \gg p_{\perp}^{2}$ in the scalar particle production case, one should employ the TMD factorization and the HEF (or CGC in the dense medium region) jointly $[8,9]$. The soft parts in the two frameworks can be treated in an unified way as shown 
above. Now we turn to discuss the perturbatively calculable hard coefficients appears in the TMD factorization and the HEF. The impact factor is calculated with the off shell incoming gluons in the HEF framework, while the hard part from the TMD factorization is estimated with on shell gluons. When $M^{2} \gg p_{\perp}^{2}$, it is justified to Taylor expand the impact fact in terms of the power $l_{\perp} / M$ where $l_{\perp}$ is the incoming gluon transverse momentum. It is easy to verify that the non-vanishing leading term of such power expansion is identical to the hard part appears in the TMD factorization formula using the Ward identity argument $[5,57-$ 59]. The similar equivalence between the TMD and the CGC has also been established for the dense medium region case $[20,21,29,31]$.

There are a number of directions in which our work could be extended. First, the natural next step is to incorporate the saturation effect [12, 13, 13-16]. Second, it should be feasible to apply the same analysis to the polarized cases. This is because the linearly polarized gluon TMD and the dipole type T-odd gluon TMDs inside a transversely polarized target have been found to grow very rapidly towards small $x$ as well $[22,23,36-38]$. We anticipate that two different type large logarithms also show up at the same time for these polarization dependent gluon TMDs in the small $x$ limit. However, we would like to emphasize that the small $x$ evolution of the gluon helicity distribution has to be treated in a complete different way $[17,18,47]$. Third, by extracting the large logarithm terms from the complete two loop order results for TMDs [60,61] one should be able to reproduce the same result presented in this paper. Finally, we have demonstrated that the Ji-Ma-Yuan scheme is compatible with the small $x$ formalism. Though there is no reason to doubt that other schemes are not compatible with the small $x$ formalism, it would be nice to check them through explicit calculations.

\section{Acknowledgments}

I thank Feng Yuan, Hsiang-nan Li, Yu-ming Wang, Miguel G. Echevarria, and Tomas Kasemets for helpful discussions. This research has been supported by the EU "Ideas" program QWORK (contract 320389).

Open Access. This article is distributed under the terms of the Creative Commons Attribution License (CC-BY 4.0), which permits any use, distribution and reproduction in any medium, provided the original author(s) and source are credited.

\section{References}

[1] J.C. Collins and D.E. Soper, Back-To-Back Jets in QCD, Nucl. Phys. B 193 (1981) 381 [Erratum ibid. B 213 (1983) 545] [INSPIRE].

[2] J.C. Collins and D.E. Soper, Parton Distribution and Decay Functions, Nucl. Phys. B 194 (1982) 445 [INSPIRE].

[3] J.C. Collins, D.E. Soper and G.F. Sterman, Transverse Momentum Distribution in Drell-Yan Pair and $W$ and $Z$ Boson Production, Nucl. Phys. B 250 (1985) 199 [InSPIRE].

[4] S. Catani, M. Ciafaloni and F. Hautmann, High-energy factorization and small $x$ heavy flavor production, Nucl. Phys. B 366 (1991) 135 [inSPIRE]. 
[5] J.C. Collins and R.K. Ellis, Heavy quark production in very high-energy hadron collisions, Nucl. Phys. B 360 (1991) 3 [InSPIRE].

[6] E.A. Kuraev, L.N. Lipatov and V.S. Fadin, The Pomeranchuk Singularity in Nonabelian Gauge Theories, Sov. Phys. JETP 45 (1977) 199 [Zh. Eksp. Teor. Fiz. 72 (1977) 377] [INSPIRE].

[7] I.I. Balitsky and L.N. Lipatov, The Pomeranchuk Singularity in Quantum Chromodynamics, Sov. J. Nucl. Phys. 28 (1978) 822 [Yad. Fiz. 28 (1978) 1597] [InSPIRE].

[8] A.H. Mueller, B.-W. Xiao and F. Yuan, Sudakov Resummation in Small-x Saturation Formalism, Phys. Rev. Lett. 110 (2013) 082301 [arXiv:1210.5792] [INSPIRE].

[9] A.H. Mueller, B.-W. Xiao and F. Yuan, Sudakov double logarithms resummation in hard processes in the small-x saturation formalism, Phys. Rev. D 88 (2013) 114010 [arXiv: 1308.2993] [INSPIRE].

[10] A.H. Mueller, L. Szymanowski, S. Wallon, B.-W. Xiao and F. Yuan, Sudakov Resummations in Mueller-Navelet Dijet Production, JHEP 03 (2016) 096 [arXiv: 1512.07127] [INSPIRE].

[11] L.D. McLerran and R. Venugopalan, Computing quark and gluon distribution functions for very large nuclei, Phys. Rev. D 49 (1994) 2233 [hep-ph/9309289] [InSPIRE].

[12] J. Jalilian-Marian, A. Kovner, A. Leonidov and H. Weigert, The Wilson renormalization group for low x physics: Towards the high density regime, Phys. Rev. D 59 (1998) 014014 [hep-ph/9706377] [INSPIRE].

[13] E. Iancu, A. Leonidov and L.D. McLerran, Nonlinear gluon evolution in the color glass condensate. 1., Nucl. Phys. A 692 (2001) 583 [hep-ph/0011241] [InSPIRE].

[14] E. Ferreiro, E. Iancu, A. Leonidov and L. McLerran, Nonlinear gluon evolution in the color glass condensate: II, Nucl. Phys. A 703 (2002) 489 [hep-ph/0109115] [INSPIRE].

[15] I. Balitsky, Operator expansion for high-energy scattering, Nucl. Phys. B 463 (1996) 99 [hep-ph/9509348] [INSPIRE].

[16] Y.V. Kovchegov, Small-x $F_{2}$ structure function of a nucleus including multiple Pomeron exchanges, Phys. Rev. D 60 (1999) 034008 [hep-ph/9901281] [InSPIRE].

[17] J. Bartels, B.I. Ermolaev and M.G. Ryskin, Nonsinglet contributions to the structure function g1 at small $x, Z$. Phys. C 70 (1996) 273, hep-ph/9507271 [INSPIRE].

[18] J. Bartels, B.I. Ermolaev and M.G. Ryskin, Flavor singlet contribution to the structure function G(1) at small x, Z. Phys. C 72 (1996) 627 [hep-ph/9603204] [INSPIRE].

[19] D. Boer, A. Dumitru and A. Hayashigaki, Single transverse-spin asymmetries in forward pion production at high energy: Incorporating small-x effects in the target, Phys. Rev. D 74 (2006) 074018 [hep-ph/0609083] [INSPIRE].

[20] F. Dominguez, B.-W. Xiao and F. Yuan, $k_{t}$-factorization for Hard Processes in Nuclei, Phys. Rev. Lett. 106 (2011) 022301 [arXiv:1009.2141] [INSPIRE].

[21] F. Dominguez, C. Marquet, B.-W. Xiao and F. Yuan, Universality of Unintegrated Gluon Distributions at small x, Phys. Rev. D 83 (2011) 105005 [arXiv:1101.0715] [INSPIRE].

[22] A. Metz and J. Zhou, Distribution of linearly polarized gluons inside a large nucleus, Phys. Rev. D 84 (2011) 051503 [arXiv:1105.1991] [INSPIRE]. 
[23] F. Dominguez, J.-W. Qiu, B.-W. Xiao and F. Yuan, On the linearly polarized gluon distributions in the color dipole model, Phys. Rev. D 85 (2012) 045003 [arXiv:1109.6293] [INSPIRE].

[24] Z.-B. Kang and F. Yuan, Single Spin Asymmetry Scaling in the Forward Rapidity Region at RHIC, Phys. Rev. D 84 (2011) 034019 [arXiv:1106.1375] [inSPIRE].

[25] Z.-B. Kang and B.-W. Xiao, Sivers asymmetry of Drell-Yan production in small-x regime, Phys. Rev. D 87 (2013) 034038 [arXiv:1212.4809] [INSPIRE].

[26] Y.V. Kovchegov and M.D. Sievert, A New Mechanism for Generating a Single Transverse Spin Asymmetry, Phys. Rev. D 86 (2012) 034028 [Erratum ibid. D 86 (2012) 079906] [arXiv: 1201.5890] [INSPIRE].

[27] Y.V. Kovchegov and M.D. Sievert, Sivers function in the quasiclassical approximation, Phys. Rev. D 89 (2014) 054035 [arXiv: 1310.5028] [INSPIRE].

[28] Y.V. Kovchegov and M.D. Sievert, Calculating TMDs of a Large Nucleus: Quasi-Classical Approximation and Quantum Evolution, Nucl. Phys. B 903 (2016) 164 [arXiv:1505.01176] [INSPIRE].

[29] E. Akcakaya, A. Schäfer and J. Zhou, Azimuthal asymmetries for quark pair production in pA collisions, Phys. Rev. D 87 (2013) 054010 [arXiv:1208.4965] [INSPIRE].

[30] T. Altinoluk, N. Armesto, G. Beuf, M. Martínez and C.A. Salgado, Next-to-eikonal corrections in the CGC: gluon production and spin asymmetries in pA collisions, JHEP 07 (2014) 068 [arXiv:1404.2219] [INSPIRE].

[31] P. Kotko, K. Kutak, C. Marquet, E. Petreska, S. Sapeta and A. van Hameren, Improved TMD factorization for forward dijet production in dilute-dense hadronic collisions, JHEP 09 (2015) 106 [arXiv: 1503.03421] [INSPIRE].

[32] A. Schäfer and J. Zhou, Process dependent nuclear $k_{\perp}$ broadening effect, Phys. Rev. D 88 (2013) 074012 [arXiv: 1305. 5042] [INSPIRE].

[33] A. Schäfer and J. Zhou, Transverse single spin asymmetry in direct photon production in polarized pA collisions, Phys. Rev. D 90 (2014) 034016 [arXiv: 1404.5809] [InSPIRE].

[34] A. Schäfer and J. Zhou, Color entanglement for $\gamma$-jet production in polarized $p A$ collisions, Phys. Rev. D 90 (2014) 094012 [arXiv:1406.3198] [InSPIRE].

[35] J. Zhou, Transverse single spin asymmetry in Drell-Yan production in polarized $p A$ collisions, Phys. Rev. D 92 (2015) 014034 [arXiv:1502.02457] [INSPIRE].

[36] A. Schäfer and J. Zhou, A note on the scale evolution of tri-gluon correlations, arXiv: 1308.4961 [INSPIRE].

[37] J. Zhou, Transverse single spin asymmetries at small $x$ and the anomalous magnetic moment, Phys. Rev. D 89 (2014) 074050 [arXiv: 1308.5912] [inSPIRE].

[38] D. Boer, M.G. Echevarria, P. Mulders and J. Zhou, Single spin asymmetries from a single Wilson loop, Phys. Rev. Lett. 116 (2016) 122001 [arXiv:1511.03485] [INSPIRE].

[39] H.-n. Li and J.-L. Lim, Soft gluons in logarithmic summations, Eur. Phys. J. C 10 (1999) 319 [hep-ph/9903267] [INSPIRE].

[40] I. Balitsky and A. Tarasov, Rapidity evolution of gluon TMD from low to moderate $x$, JHEP 10 (2015) 017 [arXiv: 1505.02151] [INSPIRE]. 
[41] I. Balitsky and A. Tarasov, Gluon TMD in particle production from low to moderate $x$, arXiv: 1603.06548 [INSPIRE].

[42] S. Forte and C. Muselli, High energy resummation of transverse momentum distributions: Higgs in gluon fusion, JHEP 03 (2016) 122 [arXiv: 1511.05561] [INSPIRE].

[43] S. Marzani, Combining $Q_{T}$ and small-x resummations, Phys. Rev. D 93 (2016) 054047 [arXiv: 1511.06039] [INSPIRE].

[44] H.-n. Li, Unification of the $k_{T}$ and threshold resummations, Phys. Lett. B 454 (1999) 328 [hep-ph/9812363] [INSPIRE].

[45] E. Laenen, G.F. Sterman and W. Vogelsang, Recoil and threshold corrections in short distance cross-sections, Phys. Rev. D 63 (2001) 114018 [hep-ph/0010080] [INSPIRE].

[46] H.-N. Li, Y.-L. Shen and Y.-M. Wang, Resummation of rapidity logarithms in B meson wave functions, JHEP 02 (2013) 008 [arXiv:1210.2978] [INSPIRE].

[47] Y.V. Kovchegov, D. Pitonyak and M.D. Sievert, Helicity Evolution at Small-x, JHEP 01 (2016) 072 [arXiv: 1511.06737] [INSPIRE].

[48] P.J. Mulders and J. Rodrigues, Transverse momentum dependence in gluon distribution and fragmentation functions, Phys. Rev. D 63 (2001) 094021 [hep-ph/0009343] [InSPIRE].

[49] X.-d. Ji, J.-p. Ma and F. Yuan, QCD factorization for semi-inclusive deep-inelastic scattering at low transverse momentum, Phys. Rev. D 71 (2005) 034005 [hep-ph/0404183] [INSPIRE].

[50] X.-d. Ji, J.-P. Ma and F. Yuan, Transverse-momentum-dependent gluon distributions and semi-inclusive processes at hadron colliders, JHEP 07 (2005) 020 [hep-ph/0503015] [INSPIRE].

[51] M.G. Echevarria, A. Idilbi and I. Scimemi, Factorization Theorem For Drell-Yan At Low $q_{T}$ And Transverse Momentum Distributions On-The-Light-Cone, JHEP 07 (2012) 002 [arXiv:1111.4996] [INSPIRE].

[52] M.G. Echevarria, T. Kasemets, P.J. Mulders and C. Pisano, QCD evolution of (un)polarized gluon TMDPDFs and the Higgs $q_{T}$-distribution, JHEP 07 (2015) 158 [arXiv:1502.05354] [INSPIRE].

[53] J. Collins, Foundations of perturbative QCD, in Cambridge monographs on particle physics, nuclear physics and cosmology, volume 32, Cambridge University Press, Cambrdige U.K. (2011).

[54] H.-n. Li and Y.-M. Wang, Non-dipolar Wilson links for transverse-momentum-dependent wave functions, JHEP 06 (2015) 013 [arXiv:1410.7274] [INSPIRE].

[55] Y.V. Kovchegov and E. Levin, Quantum Chromodynamics at High Energy, in Cambridge Monographs on particle physics, Nuclear Physics and Cosmology, volume 33, Cambrdige University Press, Cambrdige U.K. (2012).

[56] S. Fleming, The role of Glauber exchange in soft collinear effective theory and the Balitsky-Fadin-Kuraev-Lipatov Equation, Phys. Lett. B 735 (2014) 266 [arXiv:1404.5672] [INSPIRE].

[57] H. Eguchi, Y. Koike and K. Tanaka, Twist-3 Formalism for Single Transverse Spin Asymmetry Reexamined: Semi-Inclusive Deep Inelastic Scattering, Nucl. Phys. B 763 (2007) 198 [hep-ph/0610314] [INSPIRE]. 
[58] J.C. Collins and T.C. Rogers, The Gluon Distribution Function and Factorization in Feynman Gauge, Phys. Rev. D 78 (2008) 054012 [arXiv:0805.1752] [INSPIRE].

[59] Z.-t. Liang, X.-N. Wang and J. Zhou, Multiple parton scattering in nuclei: Gauge invariance, Nucl. Phys. A 819 (2009) 79 [arXiv:0805.4030] [InSPIRE].

[60] T. Gehrmann, T. Luebbert and L.L. Yang, Calculation of the transverse parton distribution functions at next-to-next-to-leading order, JHEP 06 (2014) 155 [arXiv:1403.6451] [INSPIRE].

[61] M.G. Echevarria, I. Scimemi and A. Vladimirov, Transverse momentum dependent fragmentation function at next-to-next-to-leading order, Phys. Rev. D 93 (2016) 011502 [arXiv: 1509. 06392] [INSPIRE]. 